\title{
Mitochondrial Impairment in Cerebrovascular Endothelial Cells is Involved in the Correlation between Body Temperature and Stroke Severity
}

\author{
Heng Hu ${ }^{1,2}$, Danielle N. Doll ${ }^{1}$, Jiahong Sun ${ }^{1}$, Sara E. Lewis ${ }^{1}$, Jeffrey H. Wimsatt ${ }^{3}$, Matthew J. \\ Kessler $^{4}$, James W. Simpkins ${ }^{1,2}$, Xuefang $\operatorname{Ren}^{1,2^{*}}$ \\ ${ }^{1}$ Department of Physiology and Pharmacology, ${ }^{2}$ Experimental Stroke Core, Center for Basic and Translational \\ Stroke Research, ${ }^{3}$ Department of Medicine, ${ }^{4}$ Office of Laboratory Animal Resources, Robert C. Byrd Health \\ Sciences Center, West Virginia University, Morgantown, West Virginia, 26506 USA
}

[Received August 1, 2015; Revised September 5, 2015; Accepted September 6, 2015]

\begin{abstract}
Stroke is the second leading cause of death worldwide. The prognostic influence of body temperature on acute stroke in patients has been recently reported; however, hypothermia has confounded experimental results in animal stroke models. This work aimed to investigate how body temperature could prognose stroke severity as well as reveal a possible mitochondrial mechanism in the association of body temperature and stroke severity. Lipopolysaccharide (LPS) compromises mitochondrial oxidative phosphorylation in cerebrovascular endothelial cells (CVECs) and worsens murine experimental stroke. In this study, we report that LPS $(0.1 \mathrm{mg} / \mathrm{kg})$ exacerbates stroke infarction and neurological deficits, in the mean time LPS causes temporary hypothermia in the hyperacute stage during 6 hours post-stroke. Lower body temperature is associated with worse infarction and higher neurological deficit score in the LPS-stroke study. However, warming of the LPS-stroke mice compromises animal survival. Furthermore, a high dose of LPS ( $2 \mathrm{mg} / \mathrm{kg})$ worsens neurological deficits, but causes persistent severe hypothermia that conceals the LPS exacerbation of stroke infarction. Mitochondrial respiratory chain complex I inhibitor, rotenone, replicates the data profile of the LPS-stroke study. Moreover, we have confirmed that rotenone compromises mitochondrial oxidative phosphorylation in CVECs. Lastly, the pooled data analyses of a large sample size $(\mathrm{n}=353)$ demonstrate that stroke mice have lower body temperature compared to sham mice within 6 hours post-surgery; the body temperature is significantly correlated with stroke outcomes; linear regression shows that lower body temperature is significantly associated with higher neurological scores and larger infarct volume. We conclude that post-stroke body temperature predicts stroke severity and mitochondrial impairment in CVECs plays a pivotal role in this hypothermic response. These novel findings suggest that body temperature is prognostic for stroke severity in experimental stroke animal models and may have translational significance for clinical stroke patients - targeting endothelial mitochondria may be a clinically useful approach for stroke therapy.
\end{abstract}

Key words: Hypothermia, Mitochondria, Stroke, Impairment, Endothelial Cells, Body Temperature, Severity

Stroke is the second leading cause of death and the leading cause of disability worldwide [1]. The prognostic influence of body temperature on acute stroke in patients has been recently reported [2]. Animal models of ischemic stroke are indispensable tools to investigate the pathophysiological mechanisms involved in ischemic brain injury and to develop novel therapies for stroke [3].
Temperature management is an important topic for experimental animal studies in stroke. In rodents, external warming resulted in aggravated neuronal damage following ischemia [4], and controlled-hypothermia robustly protects stroke outcomes after permanent and transient cerebral ischemia [5]. However, hypothermia has confounded experimental results in stroke [6], 
becuase it is unclear how body temperature is associated with stroke severity in animal models of experimental stroke, and the mechanisms are poorly understood.

Mitochondria play a pivotal role in cellular bioenergetics and cell survival, participating in a variety of cellular processes including generation of ATP, maintenance of body temperature, and regulation of apoptotic and other signaling pathways [7]. Mitochondrial damage has been documented in ischemic stroke patients $[8,9]$ and experimental stroke models $[10,11]$. Our recent study demonstrates mitochondrial dysfunction opens the blood-brain barrier (BBB) in both in vitro and in vivo models and controls BBB permeability in acute experimental stroke in mice [12].

Lipopolysaccharide (LPS), a major component of gram-negative bacteria cell wall, is broadly used as a bacterial infection mimic to induce a systemic inflammatory response [13]. LPS compromises mitochondrial oxidative phosphorylation in cerebrovascular endothelial cells and worsens murine experimental stroke [12]. High doses of LPS induce hypothermia [14, 15] and have been used in animal models of sepsis [16], but the mechanism by which LPS induces hypothermia is controversial [17].

Here, we studied LPS-exacerbated stroke and induced hypothermia, and investigated the relationship between body temperature and stroke outcomes in murine experimental stroke models. Inhibition of mitochondria with rotenone also exacerbated stroke outcomes and induced hypothermia after transient middle cerebral artery occlusion (tMCAO). Rotenone compromised mitochondrial oxidative phosphorylation in CVECs. Lastly, we pooled the data analyses from a large sample size $(n=353)$ of body temperature and stroke outcomes and observed a strong negative linear regression between body temperature and stroke severity. These data suggest body temperature is useful for prediction of stroke severity in experimental animal stroke models and translation of significance in clinical stroke patients and may provide support for a clinically useful approach by targeting endothelial mitochondria for stroke therapy.
A

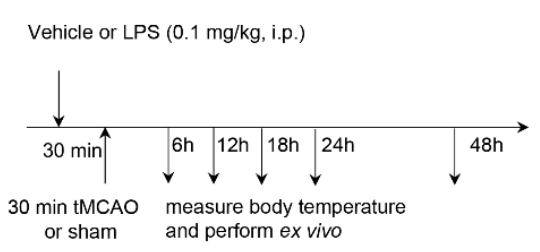

B

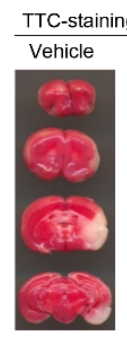

E

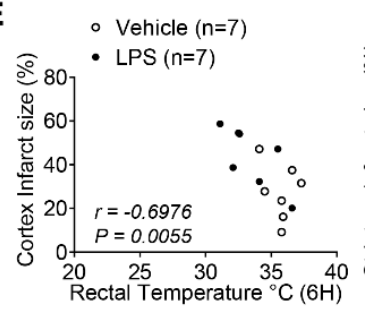

- Vehicle $(n=7)$

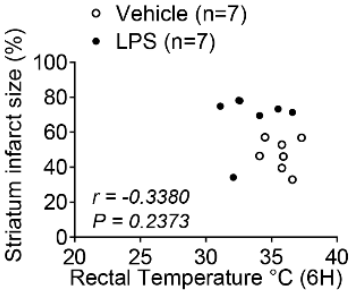

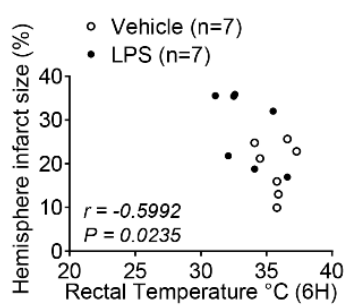

C

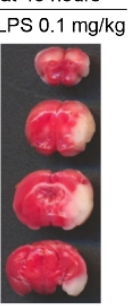

\section{.}
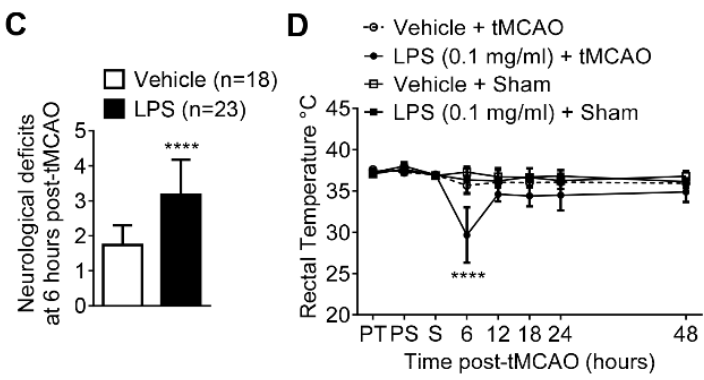

$\mathbf{F}$

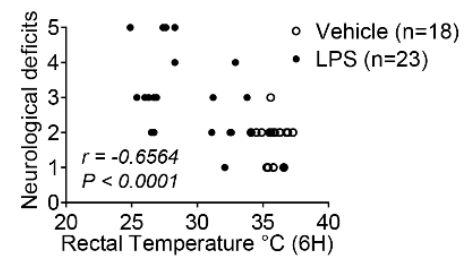

Figure 1. Low-dose LPS causes temporary hypothermia, and the body temperature is correlated with stroke outcomes. (A) Scheme of the experimental design. LPS $(0.1 \mathrm{mg} / \mathrm{kg}$, i.p.) or vehicle (saline, i.p.) was administered 30 min prior to right tMCAO (30 min occlusion) or sham and 48 hour reperfusion was performed. (B) Representative TTC-stained coronal sections from mice treated with vehicle or LPS $(0.1 \mathrm{mg} / \mathrm{kg})$, followed by $30 \mathrm{~min}$ tMCAO and 48 hours reperfusion. (C) Neurological deficits at 6 hours after tMCAO. Vehicle $=18, \mathrm{LPS}=23$; data are expressed as mean \pm S.D. $* * * *, \mathrm{P}<0.0001$; Student's $\mathrm{t}$ test. (D) Body temperature was recorded pre-treatment (PT), pre-surgery (PS), during surgery (S) and at 6, 12, 18, 24 and 48 hours. Data are expressed as mean \pm S.D.; ****, $\mathrm{P}<0.0001 ; 2$-way ANOVA followed by post-hoc Sidak's multiple comparisons test. (E) Body temperature at 6 hours post-tMCAO is correlated with \% cortex, striatum, and hemisphere infarction in 14 mice of the LPS $(0.1 \mathrm{mg} / \mathrm{kg})$ cohort of stroke study. (F) Body temperature at 6 hours post-tMCAO is correlated with neurologicial score in 41 mice of the LPS $(0.1 \mathrm{mg} / \mathrm{kg})$ cohort of stroke study. 

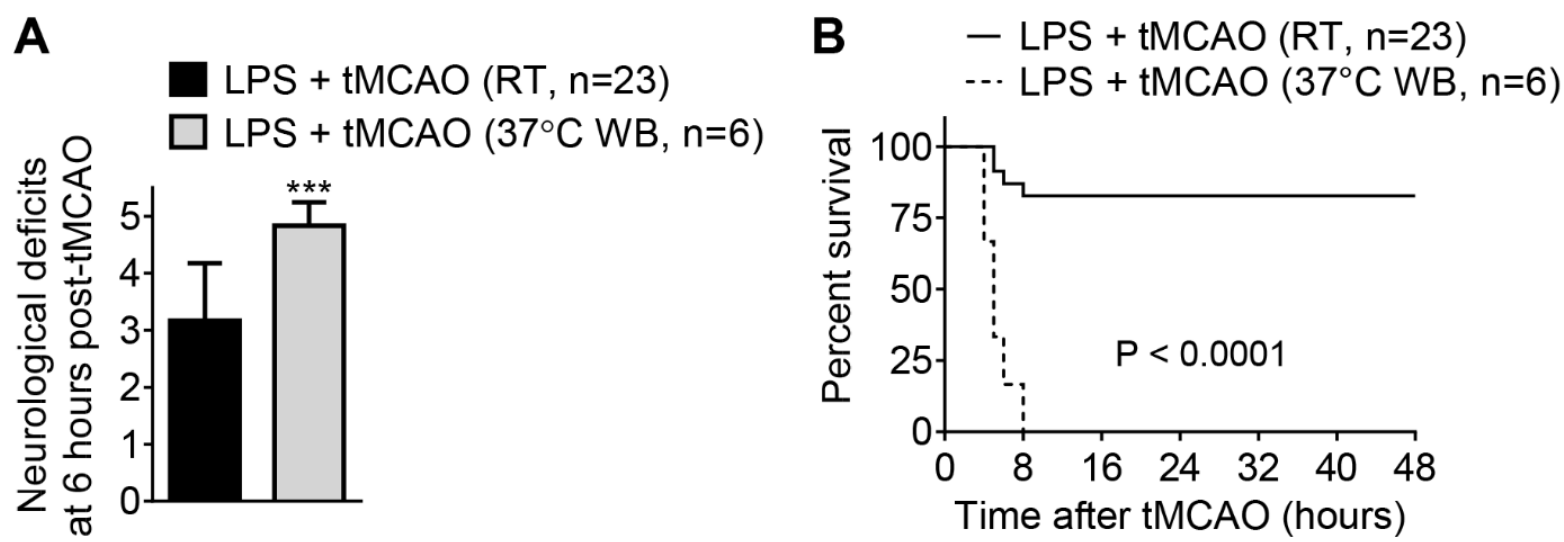

Figure 2. Rewarming worsens LPS-stroke mice and compromises animal survival. (A) External warming worsened neurological deficits of LPS-tMCAO mice at 6 hours reperfusion. Room temperature (RT) $n=23,37^{\circ} \mathrm{C}$ warm blanket (WB) $n=6$; data are expressed as mean \pm S.D. ***, $\mathrm{P}<0.001$; Student's t test. (B) External warming compromised survival of LPS-tMCAO mice. LPS (0.1 mg/kg, ip) was administered prior to right tMCAO (30 min occlusion). Post-stroke mice were housed at room temperature (RT, $\mathrm{n}=23)$ or placed on warm blanket $\left(37^{\circ} \mathrm{C}, \mathrm{n}=6\right)$. Chi square was used for statistical analysis of the survival rate.

\section{MATERIALS AND METHODS}

\section{Mice}

All procedures conducted were approved by the Institutional Animal Care and Use Committees (IACUC) at the West Virginia University (WVU). Male mice with a C57/BL6J background (3-6 months old, 25-30g, Jackson Laboratories) were used for all studies.

\section{Murine experimental stroke models}

Surgical anesthesia was induced with $4-5 \%$ isoflurane and maintained with 1-2\% isoflurane via face-mask in oxygen-enriched air. We performed focal cerebral ischemia by transient middle cerebral artery occlusion (tMCAO) or permanent middle cerebral artery occlusion (pMCAO) with a 6.0 monofilament suture (Doccol, Sharon, Massachusetts). We used laser Doppler flowmetry (Moor instruments, United Kingdom) to detect regional cerebral blood flow and confirm a successful occlusion (>70\% decrease in flow). Rectal body temperature was maintained at $37 \pm 0.5^{\circ} \mathrm{C}$ during surgery. Randomized controls were used under a common protocol that collectively assessed 353 mice, including 42 mice with sham surgery, 40 mice with pMCAO, and 271 mice with tMCAO. All surgeries were performed by one surgeon who was blinded to pre-treatments. Neurological deficits of 311 stroke mice were recorded at 6-hour, 24hour, and 48-hour end points. Infarct volume of 162 stroke mice was determined at 24-hour or 48-hour end points using 2,3,5-triphenyl-2H-tetrazolium chloride (TTC; Sigma) staining.

\section{Evaluation of neurological deficits}

Neurologic deficits were determined according to a 0-5 point scale of neurological score system as published [18]. $0=$ no neurological dysfunction; $1=$ failure to extend left forelimb fully when lifted by tail; $2=$ circling to the contralateral side; $3=$ falling to the left; $4=$ no spontaneous walk or in a comatose state; $5=$ death. The experimenters were blinded to group allocations.

\section{Drug administration}

Administration of drugs by intraperitoneal (i.p.) injection was used. The doses of LPS or rotenone were described in Results section and figure legends.

\section{Post-surgery temperature management}

Mice were housed at room temperature $\left(22-24^{\circ} \mathrm{C}\right)$ after stroke or sham surgery. Rectal temperature was measured with a rectal probe thermometer (Thermoworks, Apline, UT). External warming $\left(37^{\circ} \mathrm{C}\right.$ warm blanket) was applied to post-stroke mice in one study (Figure 2).

\section{Oxygen Consumption Rate detected by Bioscience Analyzer}

Cell culture was detailed previously [12]. Oxygen consumption rate (OCR) was measured at $37{ }^{\circ} \mathrm{C}$ using an XF96e extracellular analyzer (Seahorse Bioscience, North Billerica, Massachusetts) according to the manufacturer's instructions. Briefly, the cerebrovascular endothelial cells (bEnd.3 cell line) were seeded into Seahorse Bioscience XF96e cell culture plates $(16,000$ cells/well). After overnight culture in the $37{ }^{\circ} \mathrm{C}$ humidified incubator with 
$5 \% \mathrm{CO}_{2}$, the cells were supplied with medium rotenone (concentrations indicated in Figure 7) and cultured for additional 24 hours. For the OCR measurements, the medium was changed $1 \mathrm{~h}$ prior to the start of the extracellular flux assay to un-buffered (pH 7.4) DMEM containing $2 \mathrm{mM}$ GlutaMax, $1 \mathrm{mM}$ sodium pyruvate, and $25 \mathrm{mM}$ glucose and incubated at $37^{\circ} \mathrm{C}$ without $\mathrm{CO}_{2}$. Oligomycin, carbonyl cyanide 4-(trifluoromethoxy) phenylhydrazone (FCCP), rotenone and antimycin A (all from Sigma) of $10 \times$ compound dilutions were prepared for the assay. A sensor cartridge was loaded, calibrated and equilibrated, and then the protocol was implemented on an XF96e Analyzer. This protocol allowed determination of the basal level of oxygen consumption, the amount of oxygen consumption linked to ATP production, the maximal respiration capacity, and the non-mitochondrial oxygen consumption.

A

Vehicle or LPS $(2 \mathrm{mg} / \mathrm{kg}$, i.p.)

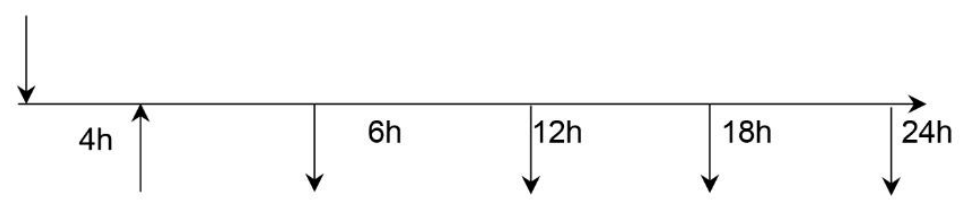

30 min TMCAO measure body temperature and perform ex vivo

B

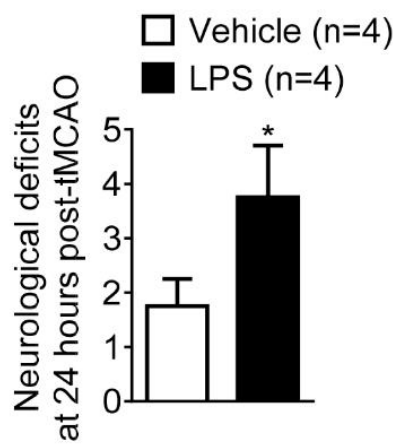

C

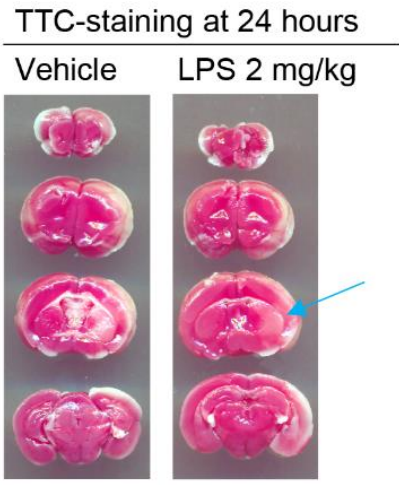

D

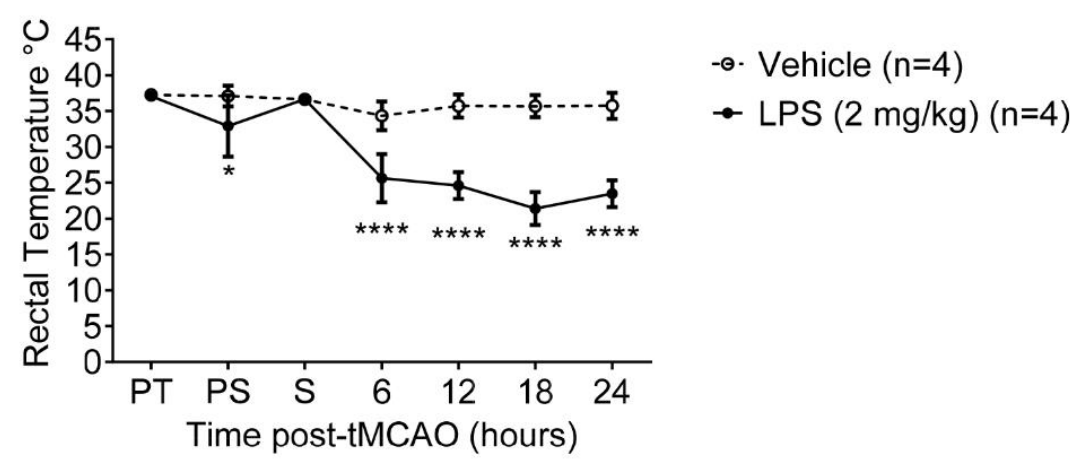

Figure 3. High-dose LPS causes persistent hypothermia, worsens neurological deficits and results in immatured brain infarction. (A) Scheme of the experimental design. LPS ( $2 \mathrm{mg} / \mathrm{kg}$, i.p.) or vehicle (saline, i.p.) was administered 30 min prior to right tMCAO (30 min occlusion) and 24 hour reperfusion was performed. (B) Neurological deficits at 24 hours after tMCAO. $\mathrm{N}=4$ per group; data are expressed as mean \pm S.D. ${ }^{*}, \mathrm{P}<0.05$; Student's $t$ test. (C) Representative TTCstained coronal sections from mice treated with vehicle or LPS $(2 \mathrm{mg} / \mathrm{kg})$, followed by $30 \mathrm{~min}$ tMCAO and 24 hours reperfusion. Blue arrow indicated immatured infarction by TTC-staining. (D) Body temperature was recorded pre-treatment (PT), pre-surgery (PS), during surgery $(\mathrm{S})$ and at 6, 12, 18 and 24 hours. Data are expressed as mean \pm S.D.; ****, $\mathrm{P}<0.0001 ; 2$-way ANOVA followed by post-hoc Sidak's multiple comparisons test. 


\section{Statistics}

Comparisons of temperature were carried out by 2-way ANOVA, followed by post-hoc Sidak's multiple comparison tests using Graphpad Prism software (Graphpad software, La Jolla, California). Student's t tests were used in analyses of statistical differences of neurological deficits between two groups. Comparisons of OCR were carried out by 1-way ANOVA followed by post-hoc Tukey's multiple comparison tests. Pearson's correlation and linear regression were used in analyses of the relationship between body temperature and stroke outcomes. Pearson's $\mathrm{r}$ value was used to establish a relationship: -0.01 to -0.19 , no or negligible relationship; -0.20 to -0.29 , weak negative relationship; -0.30 to -0.39 , moderate negative relationship; -0.40 to -0.69 , strong negative relationship; -0.70 or higher, very strong negative relationship. $\mathrm{P}<0.05$ was considered significant.
A

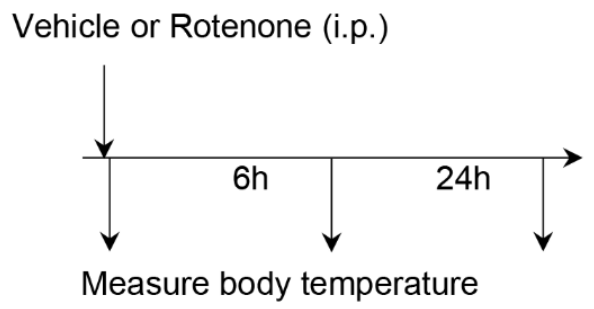

B

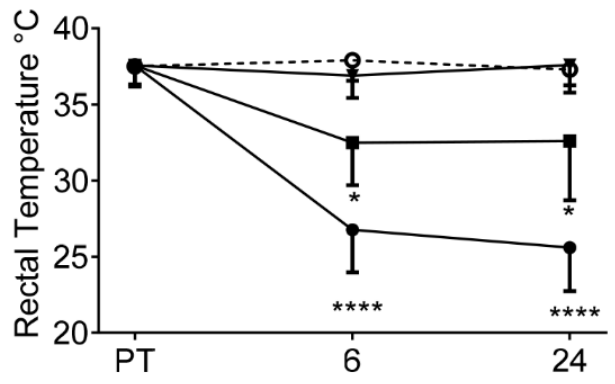

๑- Vehicle

* Rotenone $(1 \mathrm{mg} / \mathrm{kg})$

- Rotenone $(4 \mathrm{mg} / \mathrm{kg})$

$\rightarrow$ Rotenone $(10 \mathrm{mg} / \mathrm{kg})$

Figure 4. Rotenone decreases body temperature dose-dependently. (A) Scheme of the experimental design. Rotenone (i.p.) or vehicle (saline, i.p.) was administered and rectal temperature was recorded prior to treatment (PT), and at 6 and 24 hours. (B) Rotenone decreased rectal temperature in a dose-dependent manner. Data are expressed as mean \pm S.D.; *, P<0.05, ****, P<0.0001; 2-way ANOVA followed by post-hoc Sidak's multiple comparisons test.

\section{RESULTS}

\section{LPS exacerbates stroke outcomes and causes prognostic hypothermia in stroke}

We have previously reported that LPS compromises endothelial mitochondria and worsens stroke severity [12]. We applied the same study protocol as published [12] and recorded the rectal temperature as pictured in Figure 1A. LPS administration plus stroke exacerbated infarction (Figure 1B) and neurological deficits at 6 hours (Figure 1C). However, we observed that rectal temperature was significantly decreased by LPS administration plus stroke within 6 hours after injection and partially recovered after 12 hours (Figure 1D). We then performed a correlation analysis between body temperature and stroke outcomes. Interestingly, there were strong negative correlations between 6-hour body temperature and stroke outcomes including cortical infarction $(\mathrm{r}=-0.6976, P=0.0055)$ and total hemispheric infarction $(\mathrm{r}=-0.5992, \quad P=0.0235)$ (Figure 1E), and neurological score $(\mathrm{r}=-0.6564, \mathrm{P}<0.0001)$ (Figure 1F). Taken together, the data suggest that LPS exacerbates stroke outcomes and causes hypothermia, and the lower body temperature is a prognostic index of worse stroke.

It is well known that hypothermia protects stroke outcomes after permanent or transient cerebral ischemia [5]. To further investigate whether hypothermia worsens or protects outcomes in the LPS-stroke study, an external warming was applied to the LPS-stroke animals. Surprisingly, the external warming worsened neurological deficits (Figure 2A) and compromised survival of LPSstroke mice such that all mice died within 8 hours poststroke (Figure 2B). The data suggest that hypothermia does protect stroke outcomes, such an observation that seems in conflict with the observation described in Figure 1.

We have shown that LPS compromises mitochondria in CVECs dose dependently [12]. To further elucidate the confounding relationship between body temperature and stroke outcomes, a high dose of LPS (2 $\mathrm{mg} / \mathrm{kg}$ ) that induces hypothermia in mice $[14,15]$ was applied to mice following the protocol depicted in Figure $3 \mathrm{~A}$. Interestingly, a high dose of LPS plus tMCAO 
worsened neurological deficits (Figure 3B) but caused immatured stroke infarction, which showed pink staining using TTC (Figure 3C), and resulted in persistent severe hypothermia after tMCAO (Figure 3D). The data indicate that a high dose of LPS worsens stroke neurological deficits and leads to persistent severe hypothermia, which protects stroke infarction.
A

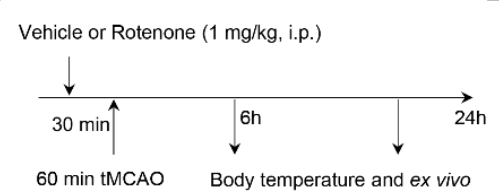

B

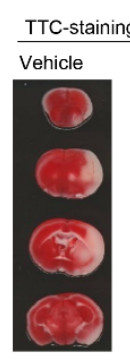

E

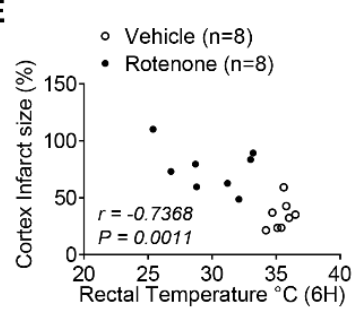

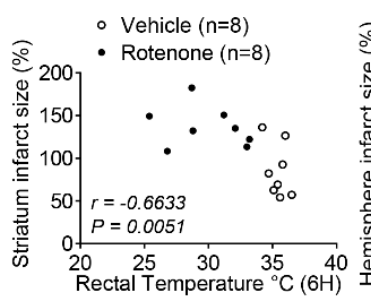
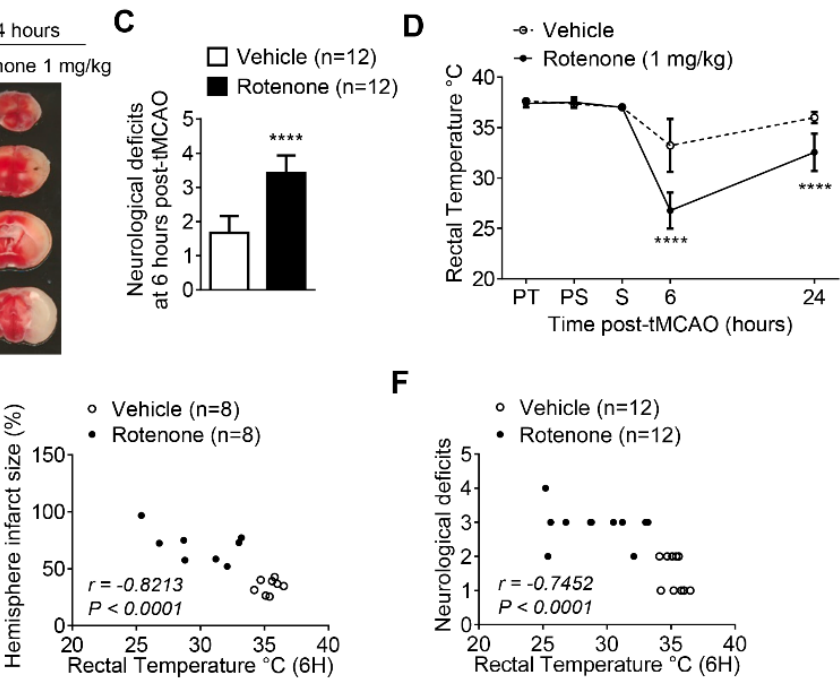

F

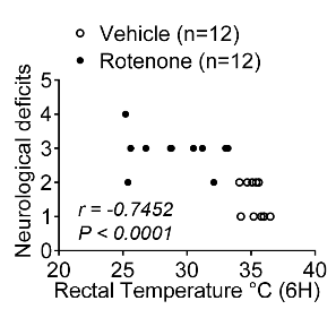

Figure. 5. Low-dose rotenone causes hypothermia, and body temperature is correlated with acute stroke outcomes in mice. (A) Scheme of the experimental design. Rotenone ( $1 \mathrm{mg} / \mathrm{kg}$, i.p.) or vehicle (saline, i.p.) was administered $30 \mathrm{~min}$ prior to right tMCAO (60 min occlusion) and 24 hour reperfusion was performed. (B) Representative TTC-stained coronal sections from mice treated with vehicle or rotenone $(1 \mathrm{mg} / \mathrm{kg}$ ), followed by $60 \mathrm{~min}$ tMCAO and 24 hours reperfusion. (C) Neurological deficits at 24 hours after tMCAO. $\mathrm{N}=12$ per group; data are expressed as mean \pm S.D. ${ }^{* * * *}, \mathrm{P}<0.0001$; Student's $\mathrm{t}$ test. (D) Body temperature was recorded pre-treatment (PT), pre-surgery (PS), during surgery (S) and at 6 and 24 hours. Data are expressed as mean \pm S.D.; $* * * *, P<0.0001$; 2-way ANOVA followed by post-hoc Sidak's multiple comparisons test. (E) Body temperature is correlated with neurologicial score in 24 mice of rotenone $(1 \mathrm{mg} / \mathrm{kg}$ ) cohort of stroke study. (F) Body temperature is correlated with \% cortex infarction, striatum infarction, and hemisphere infarction in 16 mice in the rotenone $(1 \mathrm{mg} / \mathrm{kg})$ cohort of stroke study.

\section{Mitochondrial impairment in cerebrovascular endothelial cells is involved in post-stroke hypothermia}

Due to the key role of mitochondria in cellular bioenergetics and metabolisms, low body temperature has been considered a symptom of mitochondrial failure [19]. LPS impairs mitochondrial oxidative phosphorylation [12] and causes hypothermia (Figure 1 and 3 ). To further elucidate whether mitochondrial oxidative phosphorylation is involved in the body temperature change in acute stroke, we treated mice with rotenone (a mitochondrial respiratory chain complex I inhibitor) and monitored rectal temperature (Figure 4A). Rotenone alone dose-dependently decreased body temperature in vivo (Figure 4B). In a study of the combination of rotenone and stroke, a low dose of rotenone $(1 \mathrm{mg} / \mathrm{kg})$, which did not induce hypothermia in naive mice (Figure 4), exacerbated infarction (Figure 5B), worsened neurological deficits (Figure 5C), as well as resulted in significant hypothermia following $60 \mathrm{~min}$ tMCAO (Figure 5C). Correlational analysis showed very strong negative correlations between 6-hour body temperature and neurological score $(\mathrm{r}=-0.7452, \mathrm{P}<0.0001$, Figure $5 \mathrm{E})$, cortical infarction $(\mathrm{r}=-0.7368, \mathrm{P}=0.0011)$ and hemisphere infarction $(\mathrm{r}=-0.8213, \mathrm{P}<0.0001)$, and strong negative correlations with striatum infarction $(\mathrm{r}=-0.6633$, $\mathrm{P}=0.0051$ ) (Figure 5F). These data are consistent with the findings in the low dose LPS-stroke study (Figure 1) and suggest that mitochondrial impairment is involved in the body temperature change in stroke. 
A Vehicle or Rotenone $(4 \mathrm{mg} / \mathrm{kg}$, i.p.)

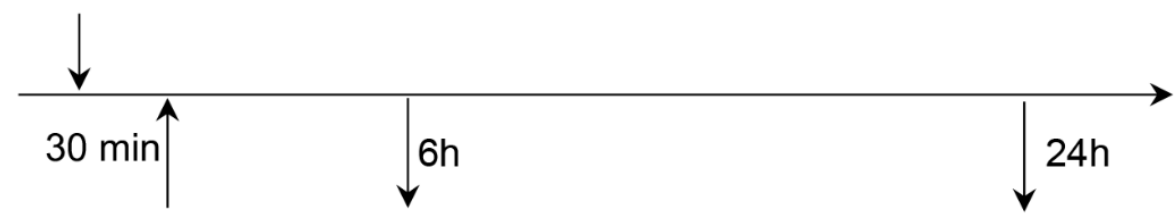

30 min tMCAO measure body temperature and perform ex vivo

B

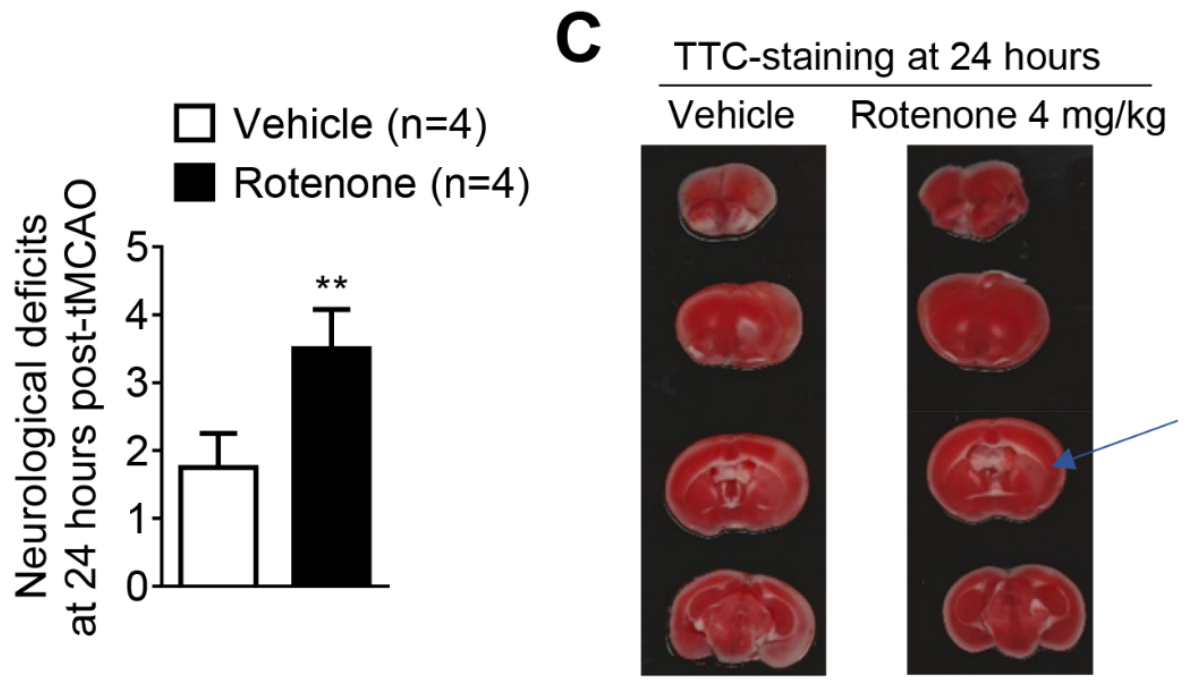

D

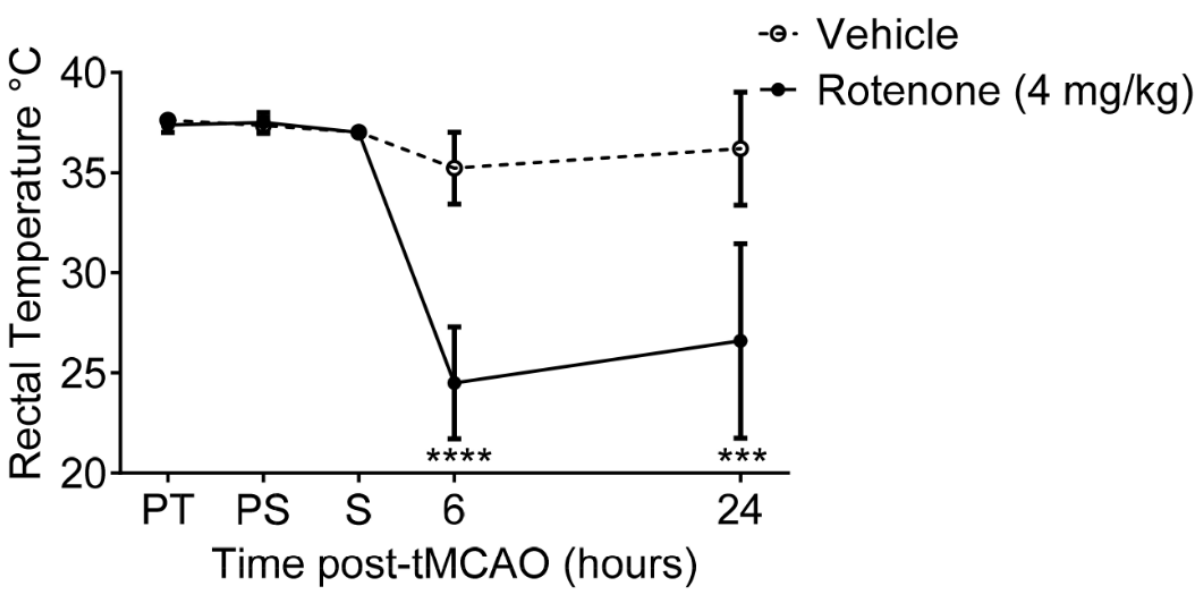

Figure 6. High-dose rotenone causes persistent hypothermia, worsens neurological deficits, and results in immatured brain infarction. (A) Scheme of the experimental design. Rotenone $(4 \mathrm{mg} / \mathrm{kg}$, i.p.) or vehicle (saline, i.p.) was administered 30 min prior to right tMCAO (30 min occlusion) and 24 hour reperfusion was performed. (B) Neurological deficits at 24 hours after tMCAO. $\mathrm{N}=4$ per group; data are expressed as mean \pm S.D. **, $\mathrm{P}<0.01$; Student's t test. (C) Representative TTC-stained coronal sections from mice treated with vehicle or rotenone $(4 \mathrm{mg} / \mathrm{kg})$, followed by $30 \mathrm{~min}$ tMCAO and 24 hours reperfusion. Blue arrow indicates immature infarction from TTC staining. (D) Rectal temperature was recorded pre-treatment (PT), pre-surgery (PS), during surgery (S) and at 6 and 24 hours. Data are expressed as mean \pm S.D.; ****, $\mathrm{P}<0.0001$, ***, $\mathrm{P}<0.001 ; 2$-way ANOVA followed by post-hoc Sidak's multiple comparisons test. 


\section{A}

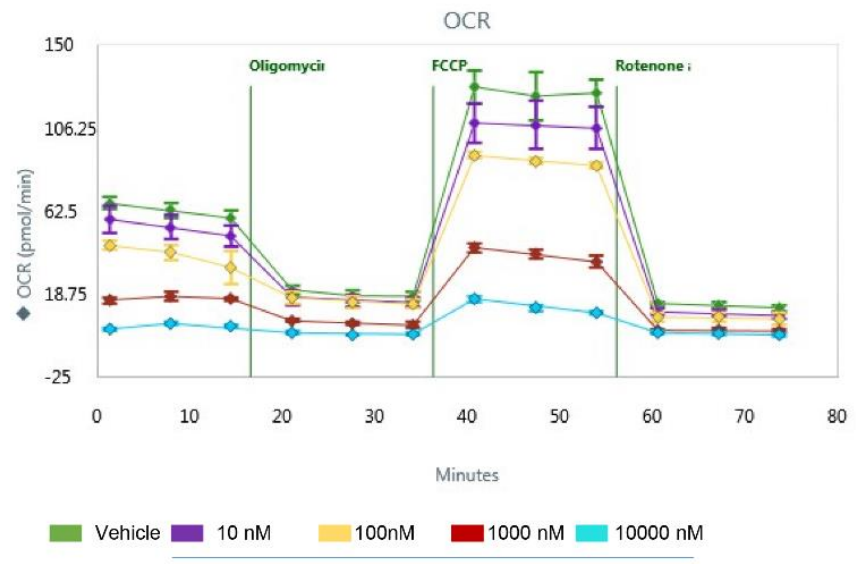

Rotenone concentration
B
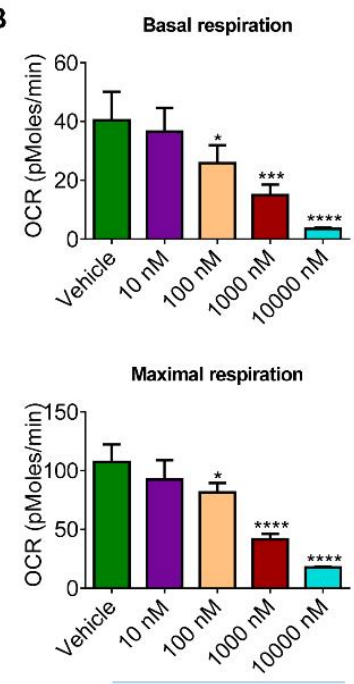

Rotenone concentration

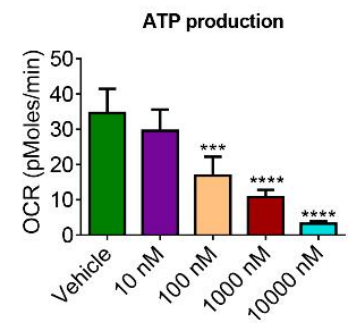

Spare capacity

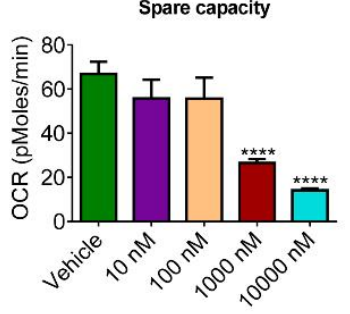

Rotenone concentration

Figure 7. Rotenone compromises mitochondrial function in cultured cerebral vascular endothelial cells. (A) Raw data of oxgen consumption rate (OCR) determinied by the Seahorse XF96e analyzer. Cultured cerebral vascular endothelial cells (bEnd3. cell line) were incubated with various concentration of rotenone for 24 hours then OCR was determined upon sequential exposure to oligomycin, FCCP, and rotenone/antimycin. (B) Analysis of the Seahorse data. Rotenone decreased basal respiration, oligomycin-sensitive ATP production, maximal respiration in the presence of FCCP, and spare capacity of cerebral vascular endothelial cells in a dose-dependent manner. $\mathrm{N}=4$ per group; *, $\mathrm{P}<0.05$; **, $\mathrm{P}<0.01$; ***, $\mathrm{P}<0.001$; ****, $\mathrm{P}<0.0001$. One-way ANOVA followed by post-hoc Tukey's test was used for statistical analysis.

Interestingly, a high dose of rotenone (4 mg/kg), which induces hypothermia in naive mice (Figure 4), worsened neurological deficits following 30 min tMCAO (Figure 6B) but resulted in immatured stroke infarction by TTC-staining (Figure 6C) and also caused persistent severe hypothermia (Figure 6D), which replicated the results from the high dose of LPS plus tMCAO mice (Figure 3). Again, the data indicate that a high dose of rotenone worsens stroke neurological deficits and results in persistent severe hypothermia that is protective for stroke infarction.

In order to identify whether rotenone affects cerebrovascular endothelial mitochondria, such as that LPS targets on CVECs in our previous report [12], a cerebrovascular endothelial cell culture model was employed and mitochondrial capacity was evaluated. We found that rotenone dose-dependently compromised mitochondrial oxidative phosphorylation in CVECs (Figure 7). Mitochondrial basal respiration, ATP production, maximal respiration, and spare capacity were all reduced in CVECs (Figure 7B). These data suggest that CVECs are one of the cellular targets in the mitochondrial impairment.

\section{Body temperature predicts stroke outcomes}

To further investigate the relationships between body temperature and stroke outcomes, we pooled the data from 353 mice, with 42 (11.9\%) sham surgery, 271 (76.8\%) tMCAO, 40 (11.3\%) pMCAO on stroke outcomes. Before surgery, there was no significant difference between the body temperatures of treatment groups (Figure 8A). Stroke mice had lower body temperature compared to sham mice within 6 hours post-surgery (Figure 8A). We performed the correlation analyses and linear regression analyses respectively to address the relationship of neurological score and infarct volumes with rectal temperature recorded within 6 hours post-surgery. There were significant negative correlations between 6 hours post-surgery body temperature and neurological deficits, and all infarct volumes (cortex, striatum, or total hemisphere) after MCAO (Figure 8B and C). Linear regression with neurological score as the dependent variable showed that lower body temperature was significantly associated with higher neurological score at all end points $\left(r=-0.5432, R^{2}=0.2951, P<0.0001\right.$, Figure 8B). Linear regression also showed significant associations between lower body temperature and larger 
cortex infarct volume $\left(\mathrm{r}=-0.3476, \mathrm{R}^{2}=0.1208, \mathrm{P}<0.0001\right)$, striatum infarct volume $\left(\mathrm{r}=-0.2782, \quad \mathrm{R}^{2}=0.07741\right.$, $\mathrm{P}=0.0003)$, and total hemisphere infarct volume $(\mathrm{r}=-$ $0.4124, \mathrm{R}^{2}=0.1701, \mathrm{P}<0.0001$ ) (Figure $8 \mathrm{C}$ ). These data suggest that hypothermia is a common response to stroke and predicts worse stroke outcomes in murine experimental stroke.
A

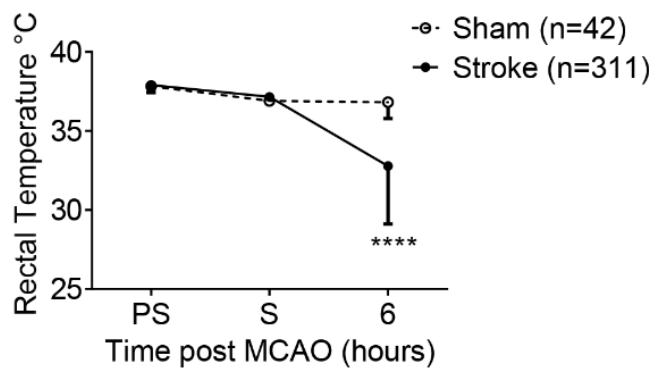

B

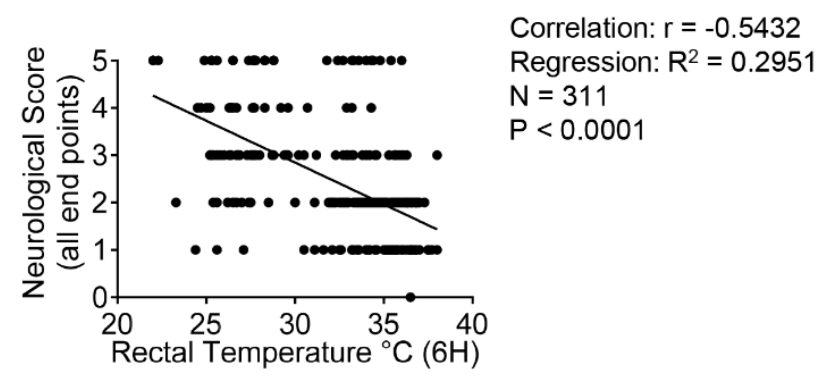

C

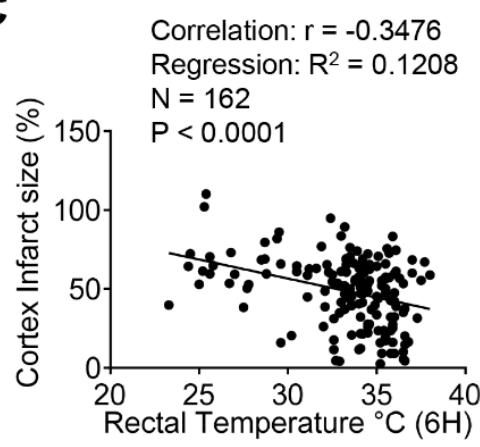

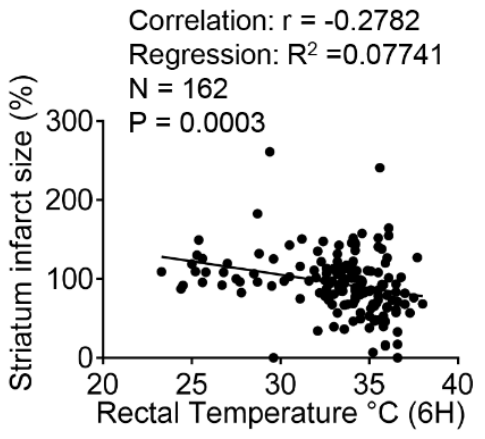

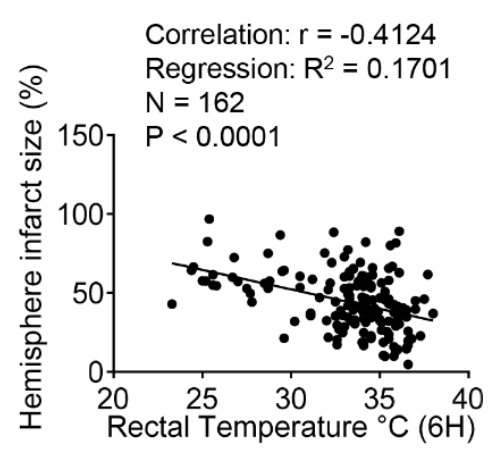

Figure 8. The relationship of body temperature and stroke outcomes in murine experimental stroke. (A) Stroke decreased body temperature. Rectal temperature was recorded pre-surgery (PS), during surgery (S), and within 6 hours post-surgery. Data are presented as mean \pm S.D.; 2-way ANOVA followed by post-hoc Sidak's multiple comparisons test. ****, P<0.0001. (B) Body temperature is correlated with neurological score in 311 mice. (C) Body temperature is correlated with \% cortex infarction, striatum infarction, and hemisphere infarction in 162 mice.

\section{DISCUSSION}

In this study, we investigated the relationship between body temperature and stroke outcomes in experimental stroke and revealed a mitochondrial mechanism in the hypothermic response to stroke. As summarized in Figure 9, stroke, LPS, or rotenone causes endothelial mitochondria impairment in the acute stage, which increases $\mathrm{BBB}$ permeability and brain edema, then worsens stroke outcomes. However, compromised endothelial mitochondria also lead to hypothermia, which delays neuronal death and protects stroke infarction, and thus conceals some of the negative effects in stroke. Therefore, hypothermia is indicative of mitochondrial impairment in $\mathrm{BBB}$ endothelium and can be used to predict stroke severity in mice.

Body temperature is one of the vital signs in monitoring medical problems. Recent clinical studies demonstrate that low body temperature within 6 hours of symptom onset is associated with severe ischemic stroke $[2,20]$ and severe stroke subjects have relatively low body temperatures at admission within 12 hours of symptom onset in human subjects [21]. In animal models of experimental stroke, it is well known that controlledhypothermia is a neuroprotectant and a potential treatment in cerebral ischemia via reduction of energy requirements in the brain and protect the brain from infarct formation $[22,23]$. Barber et al. [24] observed that temperature in 
mouse MCAO is important to stoke outcomes. Our data demonstrate a decrease in body temperature within 6 hours in experimental stroke (Figure 8). Not only did LPS- or rotenone-pretreatments reduce body temperature in experimental stroke (Fig.1D, 3D, 5D and 6D), but also in naive mice, high doses of LPS or rotenone induced hypothermic responses within hours (Figure 3D and 4B). Thus, body temperature alterations may reveal previously unknown mechanism factors that contribute to stroke severity.

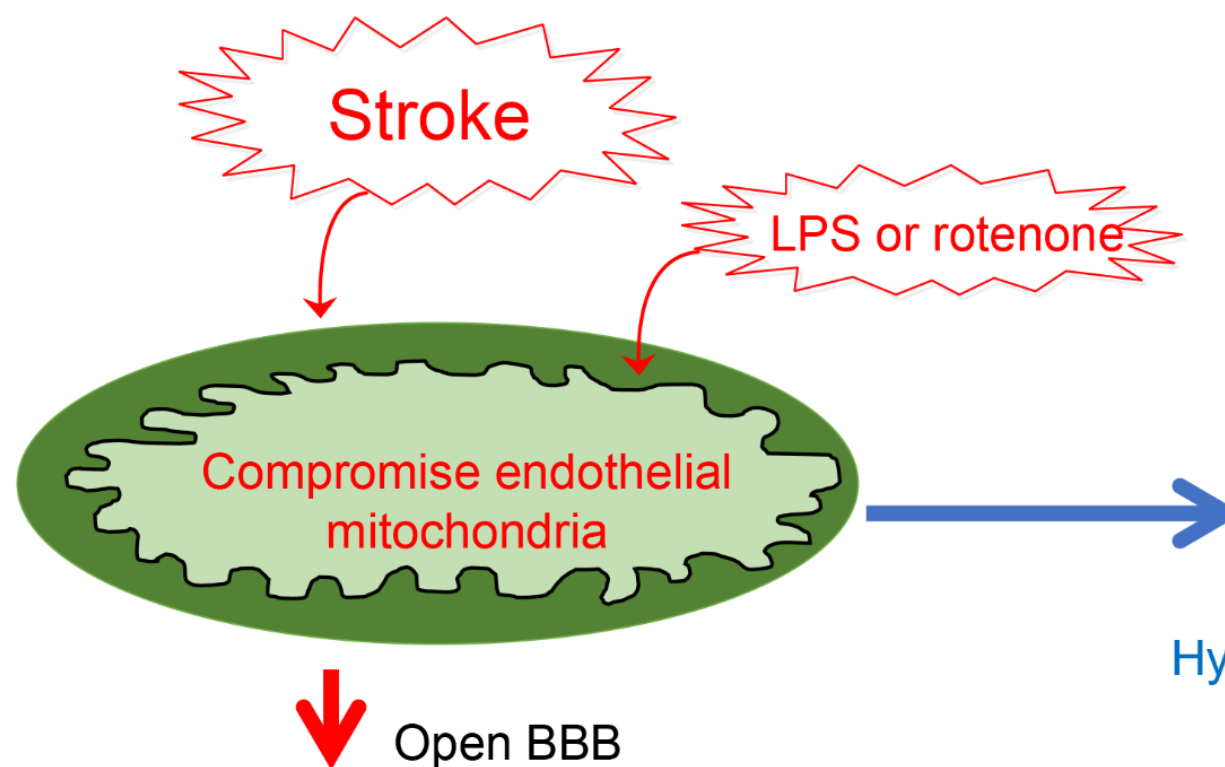

Hypothermia
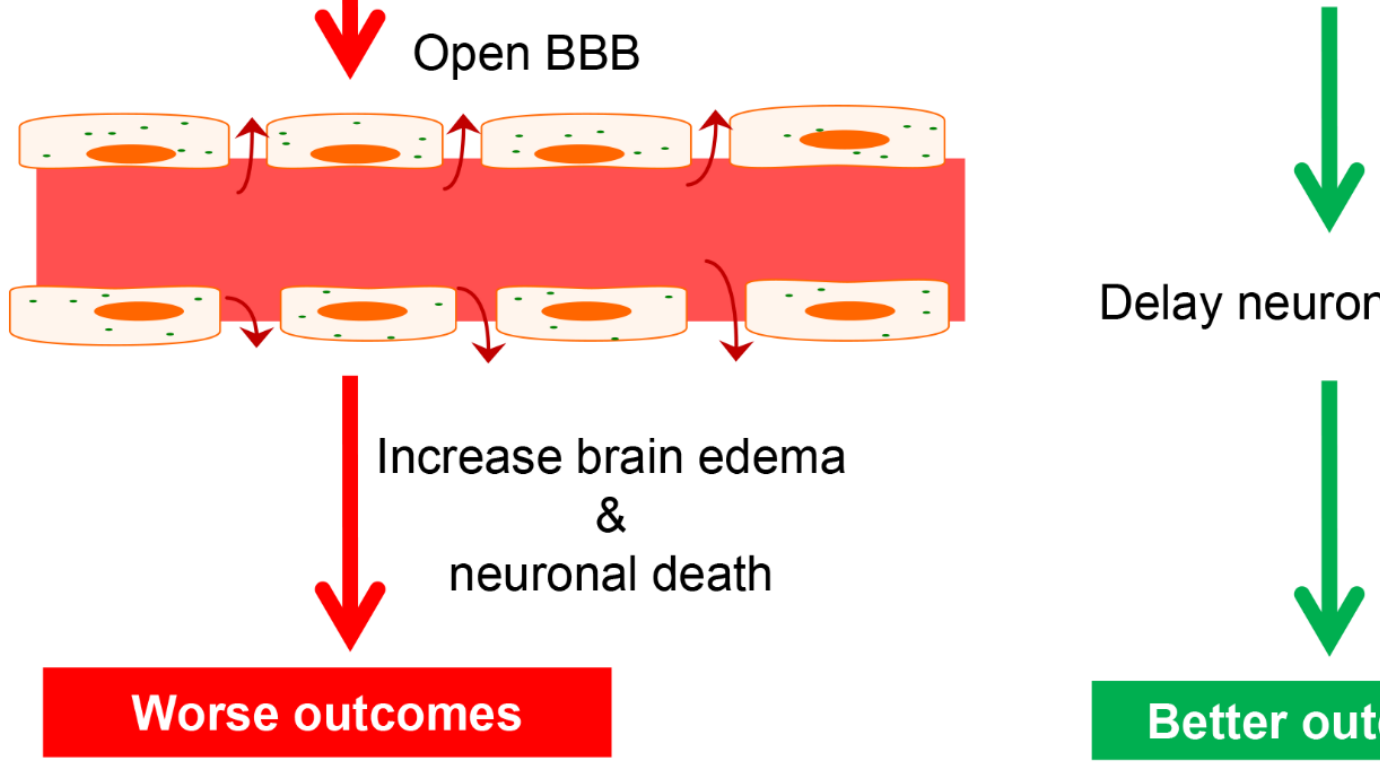

Delay neuronal death

\section{Worse outcomes}

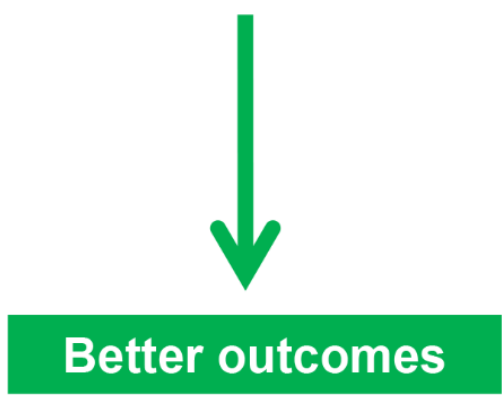

Figure 9. Endothelial mitochondria and hypothermia are engaged in stroke outcomes. Stroke, LPS or rotenone compromises endothelial mitochondria in the acute stage. The mitochondrial impairment increases BBB permeability and brain edema, further worsens stroke outcomes. The mitochondrial impairment also leads to hypothermia, which is neuroprotective and protects stroke outcomes. Hypothermia is indicative of the mitochondrial capacity and therefore has prognostic effect on stroke outcomes. 
It is known that mitochondrial oxidative metabolism is one of the homeostatic mechanisms used to generate heat for maintenance of body temperature. However, the thermoregulation is far more complex. Briefly, the vascular endothelium, smooth muscle cells, and nerve terminals form an integrated unit and regulate body temperature by preserving or releasing heat generated by mitochondrial activity in periphery and are coordinated by hypothalamus in central nervous system (CNS) [25]. Undoubtedly, the endothelium plays an important role in maintaining and regulating body temperature. Given the extensive expression of TLR4 (LPS receptor) on endothelial cells, hypothermia induced by high dose of LPS in sepsis model [26] or low dose of LPS plus tMCAO (Figure 1) may be due to compromised mitochondrial function in endothelial cells [12]. Rotenone decreased body temperature dose-dependently (Figure 4), compromised mitochondrial oxidative phosphorylation in cerebrovascular endothelial cells in vitro (Figure 3 ) and increased BBB permeability [12]. The observation of hypothermia in LPS-stroke mice (Figure 1 and 3) and rotenone-stroke mice (Figure 5 and 6), consistent with the temporal profile of BBB leakage at 6 hours post-stroke, indicates that compromised mitochondrial capacity in endothelium may not be able to maintain BBB integrity and normal body temperature, resulting in elevated BBB leakage and post-stroke hypothermia. Thus, mitochondrial mechanism may be one of the important factors of the hypothermic response to stroke. The data also suggest that the hypothermic response in severe cases of sepsis in animal studies and clinically may also be mitochondrial-dependent.

It was observed that low doses of rotenone or LPS, which did not significantly alter body temperature in sham or naive mice (Figure 1D and Figure 4), caused robust hypothermia and exacerbated stroke outcomes (Figure 1D and 5D). Both LPS [12] and rotenone (Figure 7) impair endothelial mitochondria. Stroke induces mitochondrial failure and causes cell death [27]. The low dose of LPSor rotenone may interact with stroke to further impair mitochondrial function and exacerbate stroke outcomes.

It is well-known that hypothermia protects the brain from deleterious ischemic effects and our data also confirmed its neuroprotective effect in stroke. We found that external warming compromised survival of the hypothermic LPS-tMCAO mice (Figure 2). High doses of LPS (2 mg/kg, Figure 3 ) or rotenone (4 mg/kg, Figure 6 ) could be very toxic to brain tissue, while LPS- or rotenone-induced neurotoxicity is seen with local administration [28, 29]; however, large infarctions were not observed following the combination of high doses of LPS or rotenone and tMCAO. The observed hypothermia may have protected the brain by reducing the energy and oxygen demand of brain tissue and delayed cell death in the ischemic area. Functional outcomes demonstrate sensitive or earlier indication than pathological cell death, and thus, the worse neurological deficits, rather than the stroke infarction, were observed in severe hypothermic animals (Figure 3B and 6B).

Interestingly, persistent post-stroke hypothermia resulted in immatured stroke infarction in the ischemic brain by TTC-staining and worse neurological deficits (Figure 3 and 6). These findings have been supported by other laboratories. Denes et al. [30] reported that LPS compromised survival and increased brain edema but not infarct size as assessed by crystal-violet staining; Moyer et al. [31] reported hypothermia during occlusion diminishes cerebral infarction in rats. Several groups have reported that NBQX (2,3-dihydroxy-6-nitro-7-sulfamoylbenzo (F)quinoxaline), an $\alpha$-amino-3-hydroxy-5-methyl4-isoxazole propionate antagonist, provides robust protection against neuronal loss in experimental stroke models [32-35]. However, Nurse and Corbett [36] have further verified that a hypothermic action is linearly related to the neuroprotective efficacy and NBQX is ineffective against cerebral ischemia when the body temperature was regulated to mimic that of the vehicle group. Taken together, the hypothermic response protects stroke infarction and may lead to false negative data in quantifying infarct size in experimental animal stroke studies. The false negative data could be reversed by extension of endpoints (Figure 1), lowering the dose of drugs (Figure 1 and 5), or inducing a larger injury (Figure 5). Therefore, caution should be taken in the conclusion of infarct size changes using animal models of experimental stroke.

Another important finding was the negative correlations between body temperature and stroke outcomes. In Figure 1, 5 and 8, the strong associations support that hypothermic animals have higher neurological deficits and larger infarction. The significant linear regression (Figure 8) indicates that stroke outcomes may be determined by body temperatures early after strokes. Even though the data were from pooled analyses, which might be affected by multiple factors, it does suggest that body temperatures may predict stroke outcomes. The mitochondria-dependent hypothermic response explains the correlation of hypothermia and stroke severity in vivo and the doubling of mortality rate in hypothermic sepsis patients [37]. In both disease states, induced hypothermia is thought to be protective; however, if hypothermia is a result of brain endothelial cell mitochondrial dysfunction, the association of hypothermia and exacerbated disease outcomes is not surprising because severe hypothermia indicates worse mitochondrial impairment in endothelium as described in Figure 9. These findings offer early clues on mitochondrial impairment in stroke and sepsis and offer a 
translational significance in that mitochondrial protection could be used.

The involvement of hypothalamic damage in poststroke thermoregulation has been reported [38]. In our study, only a few MCAO mice had visible hypothalamic injury (by TTC staining) with or without severe brain edema. In these few cases, injury to the hypothalamus may be caused by occlusion of hypothalamus arterial perforators, but also could be due to brain edema caused by BBB leakage. In view of the correlation between hypothermia and infarct volume as well as the rare occurrences of hypothalamic injury, we argue that the hypothermic response is due to mitochondrial impairment rather than the hypothalamic damage.

LPS [39, 40] and rotenone [41, 42] are well-known to induce systemic hemodynamic alterations. LPS may reduce interendothelial coupling and arteriolar conduction and cause hemodynamic effects [43]. Rounds and McMurtry [44] demonstrated that inhibition of ATP production by rotenone decreases vasoconstriction, blocks subsequent pressor responses, and causes hemodynamic effects. Our data demonstrated that LPS [12] or rotenone (Figure 7) compromises endothelial mitochondrial oxidative phosphorylation. Mitochondria by themselves generate reactive oxidative species (ROS) during normal respiration, and some studies suggest that when the normal function of the electron transport chain is affected, ROS output may be augmented, thus increasing the oxidative burden of the cell and inhibit pressor responses [45, 46]. The hemodynamic effects could result from the compromised endothelial mitochondria and can potentially lead to body temperature decrease as well as worsen stroke outcomes. The hemodynamic responses could also be secondary effects resulting from the compromised mitochondria in line with previous report [47], and probably is indicative of stroke severity as well.

Our study has several limitations. First, only young male mice and ischemic stroke models were used. Second, our stroke models produced large infarcts that more closely resemble malignant infarction than the average size of human strokes [48]. Third, we did not monitor post-stroke decline in spontaneous locomotor activity, which may affect body temperature. Addition of other species, multiple strains, different ages, both genders and multiple types of experimental stroke models along with long-term functional recovery are needed to firmly establish the relationship between post-stroke body temperature alterations and stroke outcomes.

In summary, this study demonstrates a negative correlation between body temperature and stroke severity in animal models of experimental stroke, elucidates a new mitochondrial-dependent mechanism for hypothermic response in stroke, and suggests the need to protect endothelial mitochondria for stroke patients with hypothermia.

\section{Acknowledgements}

This work was supported by NIH (P20 GM109098, P01 AG027956 and U54 GM104942).

\section{References}

[1] Feigin VL, Forouzanfar MH, Krishnamurthi R, Mensah GA, Connor M, Bennett DA, et al. (2014). Global and regional burden of stroke during 19902010: findings from the Global Burden of Disease Study 2010. Lancet, 383: 245-254

[2] Kim SH, Saver JL (2015). Initial body temperature in ischemic stroke: nonpotentiation of tissue-type plasminogen activator benefit and inverse association with severity. Stroke, 46: 132-136

[3] Durukan A, Strbian D, Tatlisumak T (2008). Rodent models of ischemic stroke: a useful tool for stroke drug development. Current pharmaceutical design, 14: 359370

[4] MacLellan CL, Clark DL, Silasi G, Colbourne F (2009). Use of prolonged hypothermia to treat ischemic and hemorrhagic stroke. Journal of neurotrauma, 26: 313-323

[5] van der Worp HB, Sena ES, Donnan GA, Howells DW, Macleod MR (2007). Hypothermia in animal models of acute ischaemic stroke: a systematic review and meta-analysis. Brain : a journal of neurology, 130: 3063-3074

[6] Ginsberg MD, Busto R (1998). Combating hyperthermia in acute stroke: a significant clinical concern. Stroke; a journal of cerebral circulation, 29: 529-534

[7] West AP, Shadel GS, Ghosh S (2011). Mitochondria in innate immune responses. Nature reviews. Immunology, 11: 389-402

[8] Selakovic VM, Jovanovic MD, Mihajlovic RR, Radenovic LL (2005). Dynamics of cytochrome c oxidase activity in acute ischemic stroke. Acta neurologica Scandinavica, 111: 329-332

[9] Jemmerson R, Dubinsky JM, Brustovetsky N (2005). Cytochrome $\mathrm{C}$ release from CNS mitochondria and potential for clinical intervention in apoptosismediated CNS diseases. Antioxidants \& redox signaling, 7: 1158-1172

[10] Yenari MA, Iwayama S, Cheng D, Sun GH, Fujimura M, Morita-Fujimura Y, et al. (2002). Mild hypothermia attenuates cytochrome c release but does not alter Bcl-2 expression or caspase activation after experimental stroke. Journal of cerebral blood flow and metabolism : official journal of the International Society of Cerebral Blood Flow and Metabolism, 22: 29-38

[11] Chen Q, Lesnefsky EJ (2006). Depletion of cardiolipin and cytochrome c during ischemia increases hydrogen 
peroxide production from the electron transport chain. Free radical biology \& medicine, 40: 976-982

[12] Doll DN, Hu H, Sun J, Lewis SE, Simpkins JW, Ren $X$ (2015). Mitochondrial crisis in cerebrovascular endothelial cells opens the blood-brain barrier. Stroke; a journal of cerebral circulation, 46: 1681-1689

[13] Arenas J (2012). The role of bacterial lipopolysaccharides as immune modulator in vaccine and drug development. Endocrine, metabolic \& immune disorders drug targets, 12: 221-235

[14] Habicht GS (1981). Body temperature in normal and endotoxin-treated mice of different ages. Mechanisms of ageing and development, 16: 97-104

[15] Saito H, Sherwood ER, Varma TK, Evers BM (2003). Effects of aging on mortality, hypothermia, and cytokine induction in mice with endotoxemia or sepsis. Mechanisms of ageing and development, 124: 10471058

[16] Poli-de-Figueiredo LF, Garrido AG, Nakagawa N, Sannomiya P (2008). Experimental models of sepsis and their clinical relevance. Shock, 30 Suppl 1: 53-59

[17] Al-Saffar H, Lewis K, Liu E, Schober A, Corrigan JJ, Shibata K, et al. (2013). Lipopolysaccharide-induced hypothermia and hypotension are associated with inflammatory signaling that is triggered outside the brain. Brain, behavior, and immunity, 28: 188-195

[18] Harhausen D, Khojasteh U, Stahel PF, Morgan BP, Nietfeld W, Dirnagl U, et al. (2010). Membrane attack complex inhibitor CD59a protects against focal cerebral ischemia in mice. Journal of neuroinflammation, 7: 15

[19] Sahuquillo J, Vilalta A (2007). Cooling the injured brain: how does moderate hypothermia influence the pathophysiology of traumatic brain injury. Current pharmaceutical design, 13: 2310-2322

[20] Kvistad CE, Thomassen L, Waje-Andreassen U, Naess H (2012). Low body temperature associated with severe ischemic stroke within 6 hours of onset: The Bergen NORSTROKE Study. Vascular health and risk management, 8: 333-338

[21] den Hertog HM, van der Worp HB, van Gemert HM, Algra A, Kappelle LJ, van Gijn J, et al. (2011). An early rise in body temperature is related to unfavorable outcome after stroke: data from the PAIS study. Journal of neurology, 258: 302-307

[22] Faridar A, Bershad EM, Emiru T, Iaizzo PA, Suarez JI, Divani AA (2011). Therapeutic hypothermia in stroke and traumatic brain injury. Frontiers in neurology, 2: 80

[23] Yenari MA, Han HS (2013). Influence of therapeutic hypothermia on regeneration after cerebral ischemia. Frontiers of neurology and neuroscience, 32: 122-128

[24] Barber PA, Hoyte L, Colbourne F, Buchan AM (2004). Temperature-regulated model of focal ischemia in the mouse: a study with histopathological and behavioral outcomes. Stroke; a journal of cerebral circulation, 35: 1720-1725

[25] Wise RA, Wigley F (1994). Acute effects of misoprostol on digital circulation in patients with
Raynaud's phenomenon. The Journal of rheumatology, 21: 80-83

[26] Saia RS, Anselmo-Franci JA, Carnio EC (2008). Hypothermia during endotoxemic shock in female mice lacking inducible nitric oxide synthase. Shock, 29: 119-126

Busija DW, Katakam PV (2014). Mitochondrial mechanisms in cerebral vascular control: shared signaling pathways with preconditioning. Journal of vascular research, 51: 175-189

[28] Swarnkar S, Singh S, Sharma S, Mathur R, Patro IK, Nath C (2011). Rotenone induced neurotoxicity in rat brain areas: a histopathological study. Neuroscience letters, 501: 123-127

[29] Liu M, Bing G (2011). Lipopolysaccharide animal models for Parkinson's disease. Parkinson's disease, 2011: 327089

[30] Denes A, Ferenczi S, Kovacs KJ (2011). Systemic inflammatory challenges compromise survival after experimental stroke via augmenting brain inflammation, blood- brain barrier damage and brain oedema independently of infarct size. Journal of neuroinflammation, 8: 164

[31] Moyer DJ, Welsh FA, Zager EL (1992). Spontaneous cerebral hypothermia diminishes focal infarction in rat brain. Stroke; a journal of cerebral circulation, 23: 1812-1816

[32] Sheardown MJ, Nielsen EO, Hansen AJ, Jacobsen P, Honore T (1990). 2,3-Dihydroxy-6-nitro-7-sulfamoylbenzo(F)quinoxaline: a neuroprotectant for cerebral ischemia. Science, 247: 571-574

Buchan AM, Li H, Cho S, Pulsinelli WA (1991). Blockade of the AMPA receptor prevents CA1 hippocampal injury following severe but transient forebrain ischemia in adult rats. Neuroscience letters, 132: $255-258$

[34] Diemer NH, Jorgensen MB, Johansen FF, Sheardown M, Honore T (1992). Protection against ischemic hippocampal CA1 damage in the rat with a new nonNMDA antagonist, NBQX. Acta neurologica Scandinavica, $86: 45-49$

[35] Nellgard B, Wieloch T (1992). Postischemic blockade of AMPA but not NMDA receptors mitigates neuronal damage in the rat brain following transient severe cerebral ischemia. Journal of cerebral blood flow and metabolism : official journal of the International Society of Cerebral Blood Flow and Metabolism, 12: 2-11

[36] Nurse S, Corbett D (1996). Neuroprotection after several days of mild, drug-induced hypothermia. Journal of cerebral blood flow and metabolism : official journal of the International Society of Cerebral Blood Flow and Metabolism, 16: 474-480

[37] Kushimoto S, Gando S, Saitoh D, Mayumi T, Ogura H, Fujishima S, et al. (2013). The impact of body temperature abnormalities on the disease severity and outcome in patients with severe sepsis: an analysis from a multicenter, prospective survey of severe sepsis. Critical care, 17: R271 
[38] Reglodi D, Somogyvari-Vigh A, Maderdrut JL, Vigh S, Arimura A (2000). Postischemic spontaneous hyperthermia and its effects in middle cerebral artery occlusion in the rat. Experimental neurology, 163: 399-407

[39] Jianhui L, Rosenblatt-Velin N, Loukili N, Pacher P, Feihl F, Waeber B, et al. (2010). Endotoxin impairs cardiac hemodynamics by affecting loading conditions but not by reducing cardiac inotropism. American journal of physiology. Heart and circulatory physiology, 299: H492-501

[40] Ziegler T, Horstkotte J, Schwab C, Pfetsch V, Weinmann K, Dietzel S, et al. (2013). Angiopoietin 2 mediates microvascular and hemodynamic alterations in sepsis. The Journal of clinical investigation,

[41] Gioscia-Ryan RA, LaRocca TJ, Sindler AL, Zigler MC, Murphy MP, Seals DR (2014). Mitochondriatargeted antioxidant (MitoQ) ameliorates age-related arterial endothelial dysfunction in mice. The Journal of physiology, 592: 2549-2561

[42] Zhang A, Jia Z, Wang N, Tidwell TJ, Yang T (2011). Relative contributions of mitochondria and NADPH oxidase to deoxycorticosterone acetate-salt hypertension in mice. Kidney international, 80: 51-60

[43] Bertok L (2005). Radio-detoxified endotoxin activates natural immunity: a review. Pathophysiology : the official journal of the International Society for Pathophysiology / ISP, 12: 85-95

[44] Rounds S, McMurtry IF (1981). Inhibitors of oxidative ATP production cause transient vasoconstriction and block subsequent pressor responses in rat lungs. Circulation research, 48: 393-400

[45] Balaban RS, Nemoto S, Finkel T (2005). Mitochondria, oxidants, and aging. Cell, 120: 483-495

[46] Madamanchi NR, Runge MS (2007). Mitochondrial dysfunction in atherosclerosis. Circulation research, 100: 460-473

[47] Davies PF (2009). Hemodynamic shear stress and the endothelium in cardiovascular pathophysiology. Nature clinical practice. Cardiovascular medicine, 6: $16-26$

[48] Carmichael ST (2005). Rodent models of focal stroke: size, mechanism, and purpose. NeuroRx, 2: 396-409 